\title{
Comparative cytogenetic analysis of three species of the genus Partamona (Apidae, Meliponini)
}

\author{
Geovane Campanha GonçAlves ${ }^{1}$, Anderson Medeiros Dalbosco ${ }^{1}$, Adriane Barth ${ }^{2}$, \\ Elder Assis Miranda ${ }^{1}$, Marco Antônio Costa ${ }^{1}$ \\ ${ }^{1}$ Departamento de Ciências Biológicas, Universidade Estadual de Santa Cruz, Rodovia Jorge Amado, Km 16, Ilhéus, \\ Bahia 45662-900, Brazil \\ ${ }^{2}$ Departamento de Ensino Instituto Federal de Educação, Ciência e Tecnologia de Mato Grosso-IFMT, Rondonópolis, \\ Mato Grosso 78721-520, Brazil
}

Received 29 March 2020 - Revised 16 June 2020 - Accepted 21 July 2020

\begin{abstract}
Partamona is a genus of stingless bee composed of 33 neotropical species that occur from Mexico to Brazil. The scarcity of cytogenetic studies on Partamona limits the use of this indispensable information for the recognition of species. In this study, a comparative cytogenetic analysis of three Partamona species of controversial taxonomy was carried out. Partamona ailyae, Partamona sooretamae, and Partamona aff. helleri showed the chromosome numbers, $2 n=34$ and $n=17$. However, differences were found in the karyotypic formula of $2 \mathrm{~K}=$ $22 \mathrm{M}+12 \mathrm{SM}$ in $P$. ailyae, $2 \mathrm{~K}=20 \mathrm{M}+14 \mathrm{SM}$ in $P$. sooretamae, and $2 \mathrm{~K}=28 \mathrm{M}+6 \mathrm{SM}$ in $P$. aff. helleri. $\mathrm{CMA}_{3} /$ DAPI staining revealed heterogeneity in the composition of heterochromatin, and differences in the number of $\mathrm{CMA}_{3}{ }^{+}$signals among the species, ranging from 10 in $P$. ailyae to 18 in both $P$. sooretamae and $P$. aff. helleri . Two rDNA sites were found in both $P$. ailyae and $P$. sooretamae, whereas six sites were found in $P$. aff. helleri . Moreover, chromosomal heteromorphism was found in $P$. aff. helleri, resulting from a reciprocal translocation of a NOR region between pairs 1 and 5. Although chromosome number was the same, the karyotypes were diverse due to variations in the content and distribution of constitutive heterochromatin as well as the number and location of $18 \mathrm{~S}$ rDNA regions. These variations are useful to the cytotaxonomic distinction of the species.
\end{abstract}

\section{Partamona sooretamae / Partamona ailyae /Partamona aff. Helleri / stingless bee / molecular cytogenetics}

\section{INTRODUCTION}

Partamona (SCHWARZ, 1939) is a genus of stingless bees that occur exclusively in the Neotropical region from southern Mexico to southern Brazil. Species of this genus are found in different biomes, such as rainforests, cerrado (Brazilian savanna), caatinga (dry scrub forest in northeastern Brazil), and environments transformed by human activities. This group is diverse and uses

Corresponding author: M. Costa, costama@uesc.brA. Costa, costama@uesc.br

Manuscript editor: Klaus Hartfelder several types of substrates to build nests, the entrances of which are quite distinct among different species. In some cases, these characteristics are used for taxonomic purposes, as some species of the genus are morphologically very similar and difficult to identify (Pedro and Camargo 2003; Camargo and Pedro 2003).

According to Camargo and Pedro (2003), who described 17 of the 33 species of the genus, species of Partamona are subdivided into five groups based on morphological characters and nesting characteristics: (1) epiphytophila/bilineata, (2) testacea, (3) nigrior, (4) pearsoni, and (5) cupira. Among the species of the cupira group, Partamona helleri stands out due to a set of morphological, 
taxonomic, biogeographic, behavioral, and cytogenetic peculiarities.

Partamona ailyae has a wide distribution in Brazil, occurring in the rainforests of the southwestern Amazon region, the cerrado regions of central Brazil, and further east to the state of Piauí in the northeastern region of the country (Camargo and Pedro 2003). This bee has a dark color and very defensive behavior when threatened. In the Amazon region, its nests are usually found within active termite nests on rotting tree branches or in cavities at the base of living trees. In the cerrado and other arid regions, its nests are generally found in underground termite mounds (Camargo and Pedro 2003).

Based on mitochondrial DNA sequences and morphology, Andrade et al. (2017) reported the occurrence of Partamona ailyae in the central corridor of the Atlantic Forest in the state of Bahia. However, new samples recently collected in these locations have been morphologically identified as Partamona sooretamae, which was originally described in an area between the southern portion of the state of Espírito Santo and the extreme southern portion of Bahia (Camargo and Pedro 2003).

The phylogenetic proximity and morphological similarity between these two species generated these conflicting results, making taxonomic definition problematic. The phylogenetic hypothesis presented by Camargo and Pedro (2003) placed $P$. ailyae and $P$. sooretamae as sister groups included in the cupira group. These two species share several morphological similarities. However, the description of $P$. sooretamae was based on the sampling of floral visitors and important data on the nest structure was not originally included.

The scarcity of cytogenetic studies on Partamona limits the use of this indispensable information for the recognition of species. Although the literature describes the karyotype of some species of the genus (Brito 1998; BritoRibon et al. 1999; Brito et al. 2005; Marthe et al. 2010; Martins et al. 2014; Tosta et al. 2015), the karyotype of most species remains undescribed.

Karyotype descriptions assist not only in taxonomic recognition but also provide information that can be used in phylogenetic studies and the characterization of intrapopulation and interpopulation geographic diversity as well as intraspecific and interspecific diversity, assisting in the understanding of various evolutionary mechanisms, such as chromosomal rearrangements.

The aim of the present study was to perform new comparative cytogenetic analyses of the species Partamona ailyae from the state of Mato Grosso, Partamona sooretamae from the state of Bahia, and Partamona aff. helleri from the state of Pernambuco, Brazil.

\section{MATERIALS AND METHODS}

Three nests of Partamona sooretamae were collected: two in the municipality of Presidente Tancredo Neves $\left(13^{\circ} 28^{\prime} 15^{\prime \prime} \mathrm{S} / 39^{\circ} 22^{\prime} 49^{\prime \prime} \mathrm{W} ; 13^{\circ}\right.$ $27^{\prime} 59^{\prime \prime} \mathrm{S} / 39^{\circ} 22^{\prime} 58^{\prime \prime} \mathrm{W}$ ) and one in the municipality of Uruçuca $\left(14^{\circ} 35^{\prime} 25^{\prime \prime} \mathrm{S} / 39^{\circ} 16^{\prime} 15^{\prime \prime} \mathrm{W}\right)$, both located in the Atlantic Forest in the southern portion of the state of Bahia. Two nests of Partamona aff. helleri were collected in the city of Recife in the state of Pernambuco ( $8^{\circ} 00^{\prime} 57^{\prime \prime} \mathrm{S} / 34^{\circ} 57^{\prime} 34^{\prime \prime} \mathrm{W} ; 8^{\circ}$ $00^{\prime} 56^{\prime \prime} \mathrm{S} / 33^{\circ} 57^{\prime} 36^{\prime \prime} \mathrm{W}$ ) and one nest of Partamona ailyae was collected in the municipality of Juína $\left(11^{\circ} 25^{\prime} 39^{\prime \prime} \mathrm{S} / 58^{\circ} 42^{\prime} 11^{\prime \prime} \mathrm{W}\right)$ in the state of Mato Grosso (Amazon region) (Fig. 1). The bees were identified by Dr. Gabriel A. R. Melo, Universidade Federal do Paraná.

The chromosome preparations were obtained from brain ganglion cells in the fifth larval instar, following Imai et al. (1988). Twenty samples were prepared for each nest. After air-drying for $24 \mathrm{~h}$, the samples were submitted to cytogenetic techniques for the analysis of metaphasic chromosomes.

Conventional staining was performed with Giemsa 5\% dye in Sorensen buffer and the best metaphases were photographed under an Olympus CX-41 microscope. Staining with chromomycin A3 and DAPI (4',6-diamidino-2-phenylindol) was performed according to Schweizer (1980) and analyzed using an Olympus BX-51 epifluorescence microscope to record metaphases.

Fluorescent in situ hybridization (FISH) using rDNA18S probes was performed based on Pinkel et al. (1986) with modifications according to Martins et al. (2013). The probes were labeled by BioNick Translation (Invitrogen) and the detection was performed with streptavidin-FITC 


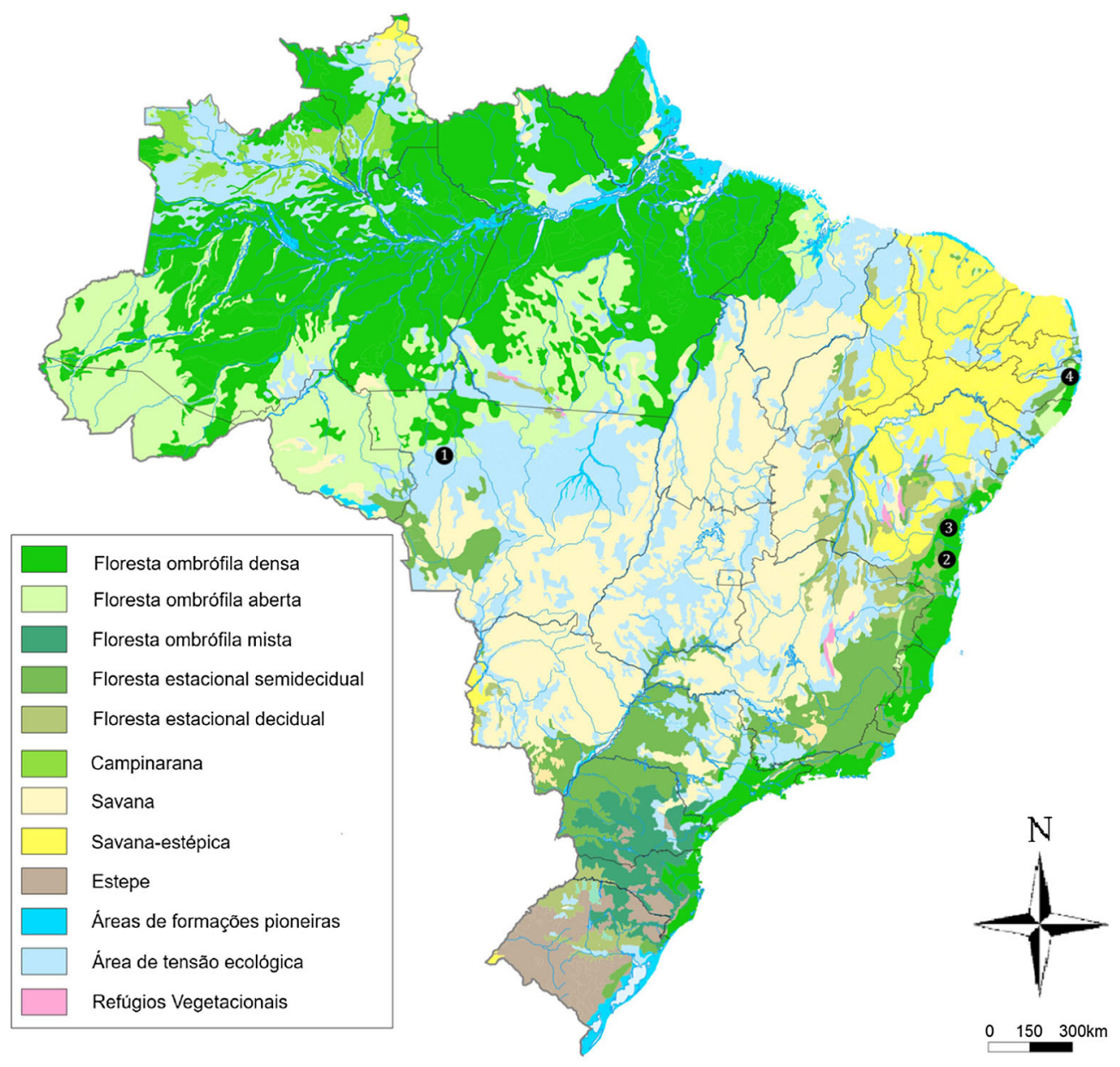

Figure 1. Map showing collection sites. (1) Juína, state of Mato Grosso, (2) Uruçuca, state of Bahia, (3) Presidente Tancredo Neves, Bahia, and (4) Recife, state of Pernambuco.

(Vector Laboratories) and streptavidin-Cy3 (Sigma-Aldrich). The images were captured in an Olympus Bx-51 epifluorescence microscope. Karyograms were organized based on Levan et al. (1964).

Ideograms were created using the DrawID software, classified in decreasing order of size and paired according to the position of the centromere.

\section{RESULTS}

Conventional staining with Giemsa revealed structural karyotype diversity. However, consistency was found in the number of chromosomes among the three species analyzed, as females had a karyotype with $2 n=34$ and males had $n=17$ (Fig. 2).

The karyotypic formula was $2 \mathrm{~K}=22 \mathrm{M}+$ $12 \mathrm{SM}$ in Partamona ailyae, $2 \mathrm{~K}=20 \mathrm{M}+14 \mathrm{SM}$ in the three colonies of Partamona sooretamae, and $2 \mathrm{~K}=28 \mathrm{M}+6 \mathrm{SM}$ in Partamona aff. helleri (Table I). Heterochromatic content was found in all chromosomes of the three species. The pairs 8 , 9, and 13 in Partamona ailyae, pairs 8, 9, and 17 in Partamona sooretamae, and pairs 12 and 17 in Partamona aff. helleri, however, were for the most part euchrochromatic (Fig. 2). The first two pairs of chromosomes were larger than the other 


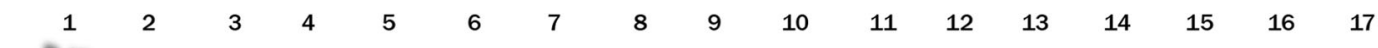 \\ If

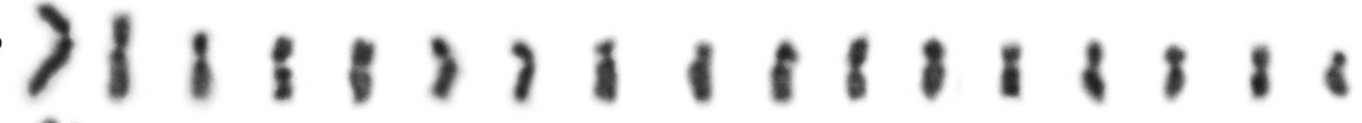

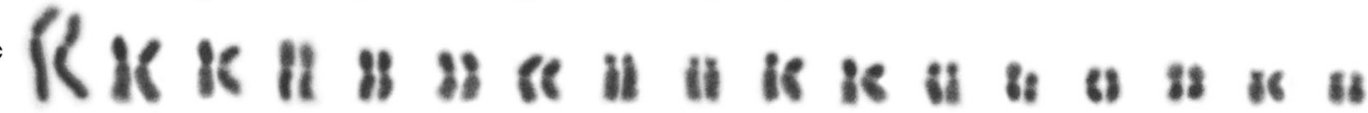

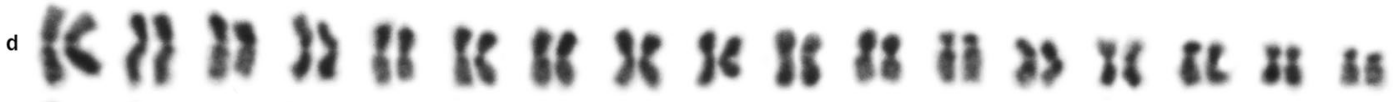 1 181818181,18181}

Figure 2. Conventional staining with 5\% Giemsa: Karyotypes of (a) female with $2 n=34$ and (b) male with $n=17$ of Partamona ailyae from Juína, Mato Grosso. (c) Partamona sooretamae female from Uruçuca, Bahia. (d) Female with $2 n=34$ and (e) male with $n=17$ from Partamona aff. helleri from Recife, Pernambuco. Bar scale: $10 \mu \mathrm{m}$.

pairs in the Partamona ailyae and Partamona sooretamae, generating a slight asymmetry in the karyotype.

Staining with $\mathrm{CMA}_{3} / \mathrm{DAPI}$ fluorochromes revealed a range of 12 to 22 chromomycin $\mathrm{A}_{3}$-positive segments $\left(\mathrm{CMA}_{3}{ }^{+}\right)$. These chromosome segments are enriched with GC base pairs. In Partamona ailyae, the chromosomes of pairs 1, 2, 3, 9, and 14 exhibited these segments. In the first pair, $\mathrm{CMA}_{3}{ }^{+}$markings were found in the terminal regions of the short arms. In the second pair, the chromosomes had two $\mathrm{CMA}_{3}{ }^{+}$regions interspersed with a narrowed $\mathrm{CMA}_{3}{ }^{-}$segments that resemble secondary constrictions. One of the chromosomes in the third pair was positively labeled throughout the long arm and in the terminal region of the short arm of the same chromosome. The ninth pair presented a large $\mathrm{CMA}_{3}{ }^{+}$marking in the terminal region of the long arms of the chromosomes. Moreover, only one chromosome had positive labeling in the terminal region of the long arm and another in the interstitial region of the short arm in pair 14 (Figs. 3a and 4a).

This is the first karyotype description of Partamona sooretamae. $\mathrm{CMA}_{3}{ }^{+}$markings were found on different chromosomes compared with those in $P$. ailyae, except for pairs 1 and 2 . In the first pair, the markings also occurred concentrated in the terminal regions of the short arms. The chromosomes of the second pair had similar markings to those of the second pair in $P$. ailyae. However, differences between the two species were exhibited in other pairs. In P. ailyae, one of the homologs in the third pair had positive

Table I. Species sampled, collection sites, karyotype formulas, $\mathrm{CMA}_{3}{ }^{+}$markings, and $18 \mathrm{~S}$ rDNA markings

\begin{tabular}{|c|c|c|c|c|}
\hline Species & Collection sites & Karyotype formulas & $\mathrm{CMA}^{+}$markings & 18S rDNA markings \\
\hline P. ailyae & Juína-MT & $2 \mathrm{~K}=22 \mathrm{M}+12 \mathrm{SM}$ & 12 & 2 \\
\hline \multirow[t]{2}{*}{ P. sooretamae } & Uruçuca-BA & $2 \mathrm{~K}=20 \mathrm{M}+14 \mathrm{SM}$ & 22 & 2 \\
\hline & Tancredo neves-BA & $2 \mathrm{~K}=20 \mathrm{M}+14 \mathrm{SM}$ & 22 & 2 \\
\hline$P$. aff. helleri & Recife-CE & $2 \mathrm{~K}=28 \mathrm{M}+6 \mathrm{SM}$ & 18 & 6 \\
\hline
\end{tabular}




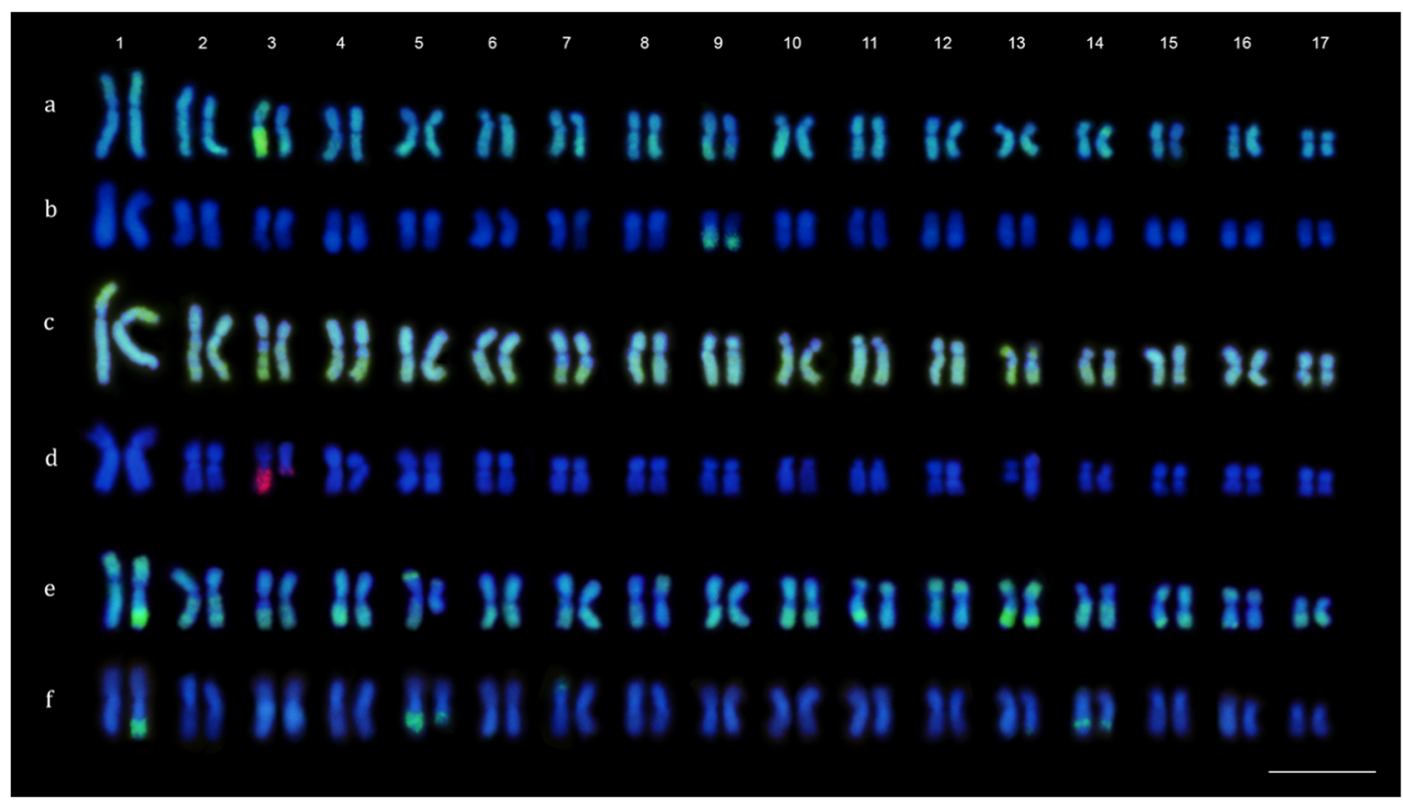

Figure 3. Karyotypes with $\mathrm{CMA}_{3}$ /DAPI staining and markings with $18 \mathrm{~S}$ rDNA probe: (a) Partamona ailyae from Juína, Mato Grosso stained with $\mathrm{CMA}_{3}$ /DAPI. (b) Partamona ailyae from Juína, Mato Grosso marked with rDNA 18S. (c) Partamona sooretamae from Uruçuca, Bahia with $\mathrm{CMA}_{3}$ /DAPI. (d) Partamona sooretamae from Uruçuca, Bahia marked with rDNA 18S. (e) Partamona aff. helleri from Recife, Pernambuco with $\mathrm{CMA}_{3} / \mathrm{DAPI}$. (f) Partamona aff. helleri from Recife, Pernambuco marked with 18S rDNA. Bar scale: $10 \mu \mathrm{m}$.

labeling in the terminal regions that were not seen in P. sooretamae. Moreover, heteromorphic $18 \mathrm{~S}$ rDNA regions were found in the third pair in P. sooretamae (Fig. 3d), differing from the labeling pattern found in P. ailyae (Fig. 3b). Other characteristics differing $P$. sooretamae from $P$. ailyae was a size heteromorphism in the 13 th pair (Figs. 2c and 3c). This size heteromorphism in $P$. sooretamae was observed in the colony of Uruçuca, BA, as well as in one of the colonies of Presidente Tancredo Neves, BA. Regarding the karyotype formula, $P$. sooretamae did not exhibit intraspecific variation in relation to two areas sampled: one in Presidente Tancredo Neves, Bahia, and another one further to the south in the state (Uruçuca, Bahia). The number of $\mathrm{CMA}_{3}{ }^{+}$ markings and $18 \mathrm{~S}$ rDNA location was consistent.

Partamona aff. helleri displayed a different pattern from the previous two species. The first pair had a conspicuous $\mathrm{CMA}_{3}{ }^{+}$marking, heteromorphic in the terminal portions of the long arms and in the terminal regions of the short arms (Figs. 3e-f and 4c). The fifth pair, which exhibited an evident $\mathrm{CMA}_{3}{ }^{+}$marking in the terminal region of the short arm of one of the chromosomes, also showed heteromorphism regarding the length of the long arm, involving a second $\mathrm{CMA}_{3}{ }^{+}$terminal region (Figs. 3e and 4c). This size heteromorphism was observed in one of the two colonies of $P$. aff. helleri sampled in Recife, PE. FISH analysis (Fig. 3f) showed that, in this case, it is caused by a variation in the length of the ribosomal clusters. One of the chromosomes in pair 11 had an interstitial $\mathrm{CMA}_{3}{ }^{+}$marking in the middle of the long arm (Figs. $3 \mathrm{e}$ and 4c). Pair 13th showed $\mathrm{CMA}_{3}{ }^{+}$markings in the terminal regions of both chromosome arms (Figs. 3e and 4c), which was similar to the pattern seen in pair 13 of $P$. sooretamae (Fig. $3 \mathrm{c}$ ) and a chromosome of 14 th pair in $P$. ailyae (Fig. 3a).

FISH using an $18 \mathrm{~S}$ ribosomal probe revealed interspecific but not intraspecific variation (Fig. 3). Partamona ailyae exhibited two markings located on the chromosomes of pair 9 (Fig. 3b). Both the colonies sampled in the state of Bahia (Uruçuca and Tancredo 
||||||||||| || || || || || || || || | || || || ||

-|| || || || || || || || || || || || || || || || ||

| || || || || | || || || || || || || || || || || ||

Figure 4. Ideograms of karyotypes stained by $\mathrm{CMA}_{3}$ /DAPI. (a) Partamona ailyae from Juína, Mato Grosso. (b) Partamona sooretamae from Uruçuca, Bahia. (c) Partamona aff. helleri from Recife, Pernambuco. DAPI ${ }^{+}$regions represented in blue and $\mathrm{CMA}_{3}{ }^{+}$regions in green. Bar scale: $10 \mu \mathrm{m}$.

Neves) were consistent in terms of the number of markings for $P$. sooretamae, which displayed two heteromorphic $18 \mathrm{~S}$ regions in the third pair (Fig. 3d). Six 18S rDNA markings were found in Partamona aff. helleri, located in four different pairs. In the first pair, only one of the chromosomes had a band, which was in the terminal region of the long arm. In the fifth pair, which was heteromorphic, the two chromosomes exhibited $18 \mathrm{~S}$ markings in the interstitial and terminal regions of the long arm. One of the chromosomes in pair 7 had a small terminal marking on the smaller arm. Although smaller, pair 14 also had regions with an $18 \mathrm{~S}$ band on both chromosomes, located in the interstitial region of the long arms (Fig. 3f).

\section{DISCUSSION}

The species studied exhibited structural differences in their karyotypes. The chromosomes were mostly metacentric and, at a smaller proportion, submetacentric. The number $2 n=34$ in females and $n=17$ in males was consistent in the three species, which demonstrates a very conserved characteristic in the regular karyotype of bees from the tribe Meliponini (Tavares et al. 2017), including several species of the genus Partamona: Partamona pearsoni, (Tarelho 1973), P. ailyae, Partamona vicina, Partamona mulata, Partamona sp. (Brito-Ribon et al. 1999), Partamona seridoensis (Brito et al. 2005), Partamona nhambiquara, Partamona chapadicola, and P. rustica (Miranda 2012).

Although supernumerary chromosomes have been described in some phylogenetically related species of Partamona, such as P. helleri (Costa et al. 1992; Martins et al. 2014) and Partamona cupira (Marthe et al. 2010), the colonies sampled in the present study showed no evidence of this phenomenon.

Consistency in the chromosome morphology was found in Partamona ailyae when we compared the data obtained herein with the results described by Brito-Ribon et al. (1999). The distribution of heterochromatin, which was found in nearly all chromosomes, also had a similar pattern, with a greater quantity concentrated in the 
short arms of most chromosomes. The only exception was the first pair, in which most of the heterochromatin was concentrated throughout the length of the long arms and, to a lesser extent, in the pericentromeric region of the short arms. Chromosomes with euchromatic regions were found in pairs 8, 9, and 13, whereas Brito-Ribon et al. (1999) found such regions in pairs 7, 10, and 16. Despite the variation in the number of chromosomes with this euchromatic pattern, all chromosomes were morphologically submetacentric.

In $P$. ailyae, both pairs 3 and 14 exhibited heteromorphism regarding the presence/ absence of $\mathrm{CMA}_{3}{ }^{+}$markings. However, observing the karyotype of $P$. aff. helleri, heteromorphism was found regarding the presence/absence of $\mathrm{CMA}_{3}{ }^{+}$markings in pairs 1, 5, 11, and 15 (Figs. 3e and 4c), which is a significant amount of heteromorphic markings in the chromosomes of a species of bee. Heteromorphic markings are common in other species of Meliponini, such as Trigona branneri (Costa et al. 2004) and the genus Scaptotrigona (Duarte et al. 2009). According to Fry and Salser (1977) cited by Sumner (2003), heteromorphisms in the composition of heterochromatin are related to differential amplifications of distinct segments that may be amplified in some sequences, forming heterochromatic blocks in some species, whereas different sequences are amplified in other species. In $P$. ailyae, two rDNA clusters were detected and were located in pair 9, in which the region corresponding to $18 \mathrm{~S}$ was heterochromatic with conventional staining (Fig. 2a and b).

The cytogenetic characterization of $P$. vicina, $P$. mulata, and P. ailyae performed by BritoRibon et al. (1999) revealed very similar results to the karyotypes of the species investigated in the present study regarding the morphology and chromosome size of the first pair. It is possible that the chromosomal homeology of this pair occurs in related species in the genus Partamona.

Andrade et al. (2017) found that specimens collected in the municipalities of Presidente Tancredo Neves, Uruçuca, and Una in the state of Bahia when compared with $P$. ailyae through
mtDNA $12 \mathrm{~S}$ analyses were possibly of the same species, as they shared an insert of five bases in this mitochondrial region in a unique way within Partamona. This hypothesis is consistent with Camargo and Pedro (2003), who present a morphological phylogeny in which these two species are closely related and placed as sister groups. However, the cytogenetic data presented herein reveal that our samples of Partamona sooretamae and Partamona ailyae exhibited differences in their karyotypes, reinforcing the idea that these may be isolated species. In case this hypothesis is correct, the sharing of the mitochondrial sequence insertion described previously would be a synapomorphy and, alternatively, be an ancestral character. A broader investigation, however, would be necessary to verify possible occurrences in other closely related species.

The karyotype of $P$. aff. helleri had the most differences compared with the karyotypes of $P$. ailyae and $P$. sooretamae. It had no similarity in size or morphology in the first pair of chromosomes and this pair also exhibited $\mathrm{CMA}_{3}$ band heteromorphism that was not found in the other species. The number of $\mathrm{CMA}_{3}{ }^{+}$markings was also high in $P$. aff. helleri, reaching 18 , which is a large number in hymenopterans. However, the chromosomes of the second pair had morphological similarities to those found in $P$. ailyae and $P$. sooretamae with regard to both morphology and $\mathrm{CMA}_{3}{ }^{+}$markings.

The present results suggest that $P$. ailyae and $P$. sooretamae are cytogenetically distinct. Although chromosome number was conserved, the karyotypes showed several structural differences due to variations in the content and distribution of constitutive heterochromatin as well as the number and location of $18 \mathrm{~S}$ rDNA regions. These differences may have important evolutionary implications and contribute to interspecific differentiation. The cytogenetic characters described, for instance, are useful to the cytotaxonomic distinction of these species.

\section{ACKNOWLEDGMENTS}

We thank Dr. Gabriel A. R. Melo for the specimen identification. 


\section{AUTHOR CONTRIBUTION}

GCG and MAC conceived this research, designed the experiments, collected samples and lab analysis, and wrote the paper. $\mathrm{AMD}, \mathrm{AB}$, and EAM collected samples, designed lab experiments, and wrote the paper.Funding information

This study was supported by Coordenadoria de Aperfeiçoamento de Pessoal de Nível Superior (CAPES), Conselho Nacional de Desenvolvimento Científico e Tecnológico (CNPq Processo: 310178/ 2015-0) and Fundação de Amparo à Pesquisa do Estado de Mato Grosso (FAPEMAT Processo: 0209383/20170).

Analyse cytogénétique comparative de trois espèces du genre Partamona (Apidae, Meliponini).

Partamona sooretamae / Partamona ailyae / Partamona aff. helleri / Abeille sans dard / Cytogénétique moléculaire.

Eine vergleichende zytogenetische Analyse für drei Arten der Stachellosen Bienen-Gattung Partamona (Apidae, Meliponini).

Partamona sooretamae / Partamona ailyae / Partamona aff. helleri / Stachellose Bienen / moleculare Zytogenetik.

\section{REFERENCES}

Andrade, M.A.P., Pedro, S.M.R., Miranda, E.A., Lama, M.A.D., Silva, M. (2017) First record of the occurrence of Partamona ailyae Camargo (Hymenoptera: Apidae) in Atlantic Forest. Sociobiology 64 (3): 363365.

Brito, R. M. (1998) Caracterização citogenética de duas espécies do gênero Partamona (Schwarz, 1939) (Hymenoptera, Apidae, Meliponinae). Dissertação de Mestrado, Universidade Federal de Viçosa, 97p.

Brito R.M., Pompolo S.G., Magalhães M.F.M., Barros E.G. and Hojo E.T.S. (2005) Cytogenetic characterization of two Partamona species (Hymenoptera, Apinae, Meliponini) by fluorochrome staining and localization of $18 \mathrm{~S}$ rDNA clusters by FISH. Cytologia 70: $373-380$.
Brito-Ribon R.M., Miyazawa C.S. and Pompolo S.G. (1999) First Karyotype Characterization of four Species of Partamona (Friese, 1980) (Hymenoptera, Apidae, Meliponini) in Mato Grosso State - Brazil. Cytobios $100: 19-26$.

Camargo, J.M.F. \& Pedro, S.R.M. (2003) Meliponini neotropicais: o gênero Partamona Schwarz, 1939 (Hymenoptera, Apidae, Apinae) - bionomia e biogeografia. Revista Brasileira de Entomologia, 47 : 311-372.

Costa, M.A., Pompolo, S.G., Campos, L.A.O. (1992) Supernumerary chromosomes in Partamona cupira (Hymenoptera, Apidae, Meliponinae). Rev. Bras. Genet. 15: 801-806.

Costa, K.F., Brito, R.M., Miyazawa, C.S. (2004) Karyotipic descrition of four species of Trigona (Jurine, 1807) (Hymenoptera, Apidae, Meliponini) from the State of Mato Grosso, Brazil. Genetics and Molecular Biology, v.27, n.2, p. 187-190.

Duarte, O.M.P., Martins, C.C.C., Waldschmidt, A.M. and Costa, M.A. (2009) Occurrence of multiple nucleolus organizer regions and intraspecific karyotype variation in Scaptotrigona xanthotricha Moure (Hymenoptera, Meliponini) Genetics and Molecular Research 8 (3): 831-839

Fry, K.; Salser, W. (1977) Nucleotide sequences of HS- $\alpha$ satellite DNA from kangaroo rat dipodomys ordii and characterization of similar sequences in other rodents. Cell v.12 n.4 p.1069-1084.

Imai, H.T., Taylor, R.W., Crosland, M.W., Crozier, R.H. (1988) Modes of spontaneous chromosomal mutation and karyotype evolution in ants with reference to the minimum interaction hypothesis. Jpn. J. Genet. 63 , 159-185.

Levan A, Fredga K., Sandberg A.A. (1964) Nomenclature for centromeric position on chromosomes. Hereditas 52: 201-220.

Marthe, J.B., Pompolo, S.G., Campos, L.A.O., Salomão, T.M.F., Tavares, M.G. (2010) Cytogenetic characterization of Partamona cupira (Hymenoptera, Apidae) by fluorochromes. Genetics and Molecular Biology, v.33, p. 253-255.

Martins, C.C.C., Diniz, D., Sobrinho-Scudeler, P.E., Foresti, F., Campos, L.A.O., Costa, M.A. (2013) Investigation of Partamona helleri (Apidae, Meliponini) $\mathrm{B}$ chromosome origin. An approach by microdissection and whole chromosome painting. Apidologie $44: 75-81$.

Martins, C.C.C., Waldschmidt, A.M., Costa, M.A. (2014) Unprecedent recordo $\mathrm{f}$ tem novel $\mathrm{B}$ chromosomes in stingless bee Partamona helleri (Apidae, Meliponini). Apidologie, $45: 431-439$.

Miranda, A.F. (2012) Estudos citogenéticos e moleculares do gênero Partamona: filogenia e cromossomos B. Tese, Universidade Federal de Viçosa.

Pedro, S.R.M. \& Camargo, J.M.F. (2003) Meliponini neotropicais: o gênero Partamona Schwarz, 1939 (Hymenoptera, Apidae). Revista Brasileira de Entomologia, $47: 1-117$. 
Pinkel, D.; Straume, T.; GRAY, J.W. (1986) Cytogenetic analysis using quantitative, high-sensitivity, fluorescence hybridization. Proc. Natl. Acad. Sci. USA 83 :2934-2938.

Schweizer, D. (1980) Simultaneous fluorescent staining of $\mathrm{R}$ bands and specific heterochromatic regions (DADAPI bands) in Human chromosomes. Cytogenet. Cell Genet. 27, 190-193.

Sumner, A.T. (2003) Chromosomes: Organization and Function. Blackwell Publishing Company, London, p. 287.

Tarelho Z.V.S. (1973) Contribuição ao estudo citogenético dos Apoidea. MS dissertation. Ribeirão Preto, FFCLRP-USP.
Tavares. M.G., Lopes, D.M., Campos, L.A.O. (2017) An overview of cytogenetics of the tribe Meliponini (Hymenoptera: Apidae). Genetica, 145 : 241-258.

Tosta, V. C., Marthe, J. B., Tavares, M. G., FernandesSalomão, T. M., Pompolo, S. G., Recco-Pimentel, S. M., Perfectti, F., Campos, L. A. O.; Camacho, J. P. M. (2015) Possible Introgression of B Chromosomes between Bee Species (Genus Partamona). Cytogenetic and Genome Research, 144, 217-223.

Publisher's note Springer Nature remains neutral with regard to jurisdictional claims in published maps and institutional affiliations. 